\title{
Morphometric Analysis of Neurons in Ganglia: Geniculate, Submandibular, Cervical Spinal and Superior Cervical
}

\author{
By
}

\author{
Hiroshi MORIYAMA, Kazuyuki SHIMADA and Noboru GOTO
}

Department of Anatomy, Showa University School of Medicine, 5-8, Hatanodai 1, Shinagawa-ku, Tokyo 142, Japan

- Received for Publication, July 20, $1995-$

\begin{abstract}
Key Words: Neuron, Morphometry, Ganglion, Human, Peripheral nerve
Summary: The geniculate ganglia (GG), the submandibular ganglion (SG), the cervical spinal ganglion (CSG) and the superior cervical ganglion (SCG) are clinically important. But details of these ganglia have not been included in textbook descriptions. We conducted research to bring precise figures to light. We prepared serial sections of these ganglia in five Japanese adults after precise dissections to count the numbers, measure the perimeters and the areas, and calculate the circularity ratios (CRs) of the ganglion cells. The numbers of neurons were in the following order: SCG $>$ CSG $>$ SG $>$ GG, and the average neuronal areas in the following order: $\mathbf{C S G}>\mathbf{G G}>\mathbf{S G}>$ SCG. Average neuronal CRs were around 0.90 in all ganglia. In other words, the morphometric evaluation showed that neurons in such ganglia are practically circular in shape. Our morphometry thus reveals that the neurons in these ganglia are sensory neurons in the broad definition of the word from a quantitative point of view.
\end{abstract}

Precise knowledge of various ganglia is very important for a thorough understanding of clinical medicine. Details of these ganglia, however, have not been included in textbook descriptions. We conducted our research to bring precise figures to light. We selected several ganglia as follows: the GG, which is the site of nerve cell bodies for the special sense of taste; the SG, which supplies preganglionic visceral efferent (secretomotor) fibers innervating the submandibular and sublingual salivary glands; the CSG and SCG, which are linked to the dorsal root and to the sympathetic trunk, respectively. We made serial sections of these ganglia in five Japanese adults after precise dissections to count the numbers, to measure the perimeters and the areas, and to calculate the CRs of the ganglion cells.

\section{Material and Methods}

The material for the GG and the SG was four head-halves from cadavers collected for anatomic dissections. After decalcification of these head-halves by Plank-Rychlo fluid, we made precise dissections of the GG and the SG under an operation microscope (Wild 630, Leica, Heerbrugg, Switzerland or OME, Olympus, Tokyo, Japan). The fifth CSG and the
SCG were prepared for histologic analysis. We counted the numbers, measured the perimeters and areas, and calculated the CRs of these ganglion cells. The precise procedures were as follows:

\section{Fixation:}

Primary fixative: $3.7 \%$ solution of formaldehyde. Secondary fixative: mixture of $5 \%$ potassium dichromate and $5 \%$ potassium chromate (1:4 in volume) for three weeks (two weeks at room temperature, followed by one week at $37^{\circ} \mathrm{C}$ ). For more details, see the reference section (Goto, 1987).

\section{Washing in running water:}

The fixed tissue blocks were washed with a siphonoperated automatic pipette washer fitted with a nylon-mesh bag or a plastic tissue-basket.

Dehydration with alcohol and celloidin embedding: Dehydration of tissue blocks started from $50 \%$ ethanol, increasing to $70,90,95 \%$ and absolute, followed by celloidin embedding.

\section{Sectioning:}

Celloidin blocks were cut into $30 \mu \mathrm{m}$ thick sections to make serially numbered sections. 


\section{Staining:}

For the GG and the SG, every fifth section was stained by LPH triple method (Goto, 1987). For the CSG and the SCG, every third section was stained by K-B method.

\section{Morphometry:}

The systemic sampling method (quasi-random sampling) was employed. The neurons having a distinct nucleolus were counted under the microscope taking into consideration Abercrombie's counting criteria for histology sections (Abercrombie, 1946). The numbers, perimeters and areas of the ganglion cells in each sampling area were measured with a combination of microscope (BH2, Olympus, Tokyo, Japan), drawing tube (BH2-DA, Olympus, Tokyo, Japan), digitizer (Crystizer $\alpha, \mathrm{KC} 3300$, Graphtec, Yokohama, Japan) and data storing and analyzing system (original programming). A computer (PC-9801 VX 2 , NEC, Tokyo, Japan) was employed for storing data on-line, calculation and statistical analysis. Circularity ratios (CRs) were calculated as follows:

$\mathrm{CR}=4 \pi \mathrm{A} / \mathrm{L}^{2}\left(\mathrm{~A}=\right.$ area in $\mathrm{mm}^{2} ; \mathrm{L}=$ perimeter in $\mathrm{mm}$ )

\section{Results}

Microscopic observation revealed that the CSG contained large neurons, while the SCG contained only small neurons (Figs. 3 and 4). Under high power viewing, the nerve cell bodies of the ganglia appeared to be rather circular in shape, containing a round nucleus with a prominent nucleolus inside, and fine Nissl bodies in the perikarya of somata. The nucleus tended to be centrally located in the CSG and GG, while it was eccentrically located in the SG and SCG (Figs. 1-4). The Nissl bodies of the CSG cells were of two types: fine powdery granules, and coarse granules. The former were distributed wholly inside the perikarya, the latter were aligned at the periphery of the perikarya. The Nissl bodies of the other ganglia cells were of medium size, and were distributed all over the cell soma. Each ganglion cell was surrounded by small satellite cells, each with a small, dark colored nucleus. The satellite cells in the SCG were fewer in numbers than those in the other ganglia. The neurons were the most numerous in the SCG, and the least numerous in the GG. With regard to average neuronal area, it was the largest in the CSG, and the smallest in the SCG. Average neuronal CRs were close to 0.90 in the all ganglia. Table 1 lists data regarding neuronal numbers, average neuronal areas and average neuronal CRs.

\section{Discussion}

There are several problems involved in morphometric analyses which need to be solved or taken into account. One of them is that paraffin-embedding causes a considerable but inconsistent shrinkage of sections (Cammermeyer, 1967). To minimize errors, we used the celloidin-embedding method after secondary fixation (Goto, 1987). The neuronal cell body, its nucleus, or its nucleolus, may be taken as a count unit. The larger the unit, and the thinner the section, the greater the chances of an error due to duplicate counting of units split by the sectioning and thus appearing in several sections (Koningsmark, 1970). We used a distinct neuronal nucleolus as count unit. The following three problems were considered to determine the section thickness:

(1) errors due to duplicate counting of nucleoli split by the sectioning should be within the limits of tolerance.

(2) staining should be suitable for counting.

(3) the section thickness should allow the taking of high power microscopic photographs.

After consideration of the above elements, we decided that the most appropriate thickness for our study should be $30 \mu \mathrm{m}$.

\section{Neuronal numbers:}

According to Schimert (1936), the number of cells in the GG is 1,400. Buskirk (1945), however, mentioned an average number of 2,129 with a range of 1,462 to 3,682 . Our average number was 2,043, approximating Buskirk's data. According to Davenport and Bothe (1934), the number of the cells in the second CSG is 49,000 and that in the sixth CSG is 60,000 . Our findings for the fifth CSG were a count of 56,199 approximating the data of Davenport and Bothe. Ebbesson $(1963,1968)$ reported that the average number of nerve cells in the SCG was 911,294

Table 1. Neuronal numbers, areas and CRs in ganglia

\begin{tabular}{rrrr}
\hline & Number & Area $\left(\mu \mathrm{m}^{2}\right)$ & CR \\
\hline GG No. 1 & 2,158 & 942 & 0.90 \\
No. 2 & 1,892 & 1,018 & 0.89 \\
No. 3 & 2,079 & 780 & 0.89 \\
Average & 2,043 & 913 & 0.89 \\
SG & 7,758 & 746 & 0.89 \\
CSG & 56,199 & 2,032 & 0.91 \\
SCG & 444,772 & 367 & 0.90 \\
\hline
\end{tabular}

GG No. 1: 93-year-old male; No. 2: 77-year-old male; No. 3: 49-year-old female; Average: the average value of No. 1, No. 2 and No. 3; SG: 93-year-old female; CSG: 60-year-old male; SCG: 60 -year-old male.

Areas and CRs show average values. $C R$ of a regular circle: 1.00 
with a range of 760,370 to $1,041,652$. Our count gave a total of 444,772 . We found an average cell density per $30 \mu \mathrm{m}$ thick section of SCG of 8,553 , while that of Ebbesson (1963) was 10,380 slightly higher than ours. But in so far as the length of the SCG in our material (below $20 \mathrm{~mm}$ ) was different from that of Ebbesson (about $30 \mathrm{~mm}, 1963,1968$ ), this could explain the difference in neuronal numbers between our data and Ebbesson's. We also compared neuronal numbers of the GG with those of the SG, CSG and SCG. They were in the following order: SCG $>$ CSG $>$ SG $>$ GG.

\section{Average neuronal areas:}

We compared average neuronal areas of the GG with those of the SG, the fifth CSG and the SCG. The average neuronal area was in the following order: $\mathrm{CSG}>\mathrm{GG}>\mathrm{SG}>\mathrm{SCG}$. The morphometric areal data of several ganglion cells have not been included in the references.

\section{Average neuronal CRs:}

We compared average neuronal CRs of the GG with those of the SG, the fifth CSG and the SCG. The CR shows how far from a regular circle the shape is, with a maximum value 1.0 for a perfect circle. A lower value indicates an irregular shape. The CRs of motor neurons may show low values. For example, the $\mathrm{CR}$ for motor trigeminal neurons is 0.4 (Hamano et al., 1988), for hypoglossal neurons 0.4 (Nara et al., 1989), and for facial neurons under 0.7 (Nara et al., 1989). On the other hands, the CRs for non-motor neurons approximate the values we found for the four kinds of ganglion cells. For example the CR for mesencephalic trigeminal neurons is 0.86 (Hamano et al., 1990), and for ventral cochlear neurons 0.81 (Nara et al., 1993). Our morphometry thus revealed that the cells in these ganglia can be considered to be sensory in a wide sense from a quantitative point of view, as their CRs were all close to 0.90 .

\section{References}

1) Abercrombie $M$. Estimation of nuclear population from microtome sections. Anat Rec 1946; 94:239-247.

2) Buskirk CV. The seventh nerve complex. J Comp Neurol 1945; 82:303-333.

3) Cammermeyer J. Artifactual displacement of neuronal nuclei in paraffin sections. J Hirnforsh 1967; 9:209-224.

4) Davenport HA and Bothe RT. Cells and fibers in spinal nerves. II. A study of $C_{2}, C_{6}, T_{4}, T_{9}, L_{3}, S_{2}$ and $S_{5}$ in man. J Comp Neurol 1934; 59:167-174.

5) Ebbesson SOE. A quantitative study of human superior cervical sympathetic ganglia. Anat Rec 1963; 146:353-356.

6) Ebbesson SOE. Quantitative studies of superior cervical sympathetic ganglia in a variety of primates including man. I. The ratio of preganglionic fibers to ganglionic neurons. J Morphol 1968; 124:117-131.

7) Ebbesson SOE. Quantitative studies of superior cervical sympathetic ganglia in a variety of primates including man. II. Neuronal packing density. .J Morphol 1968: 124: 181-185.

8) Goto N. Discriminative staining methods for the nervous system: luxol fast blue - periodic acid-Schiff - hematoxylin triple stain and subsidiary staining methods. Stain Technol 1987; 62:305-315.

9) Hamano S, Goto N and Nara T. Development of the human motor trigeminal nucleus. Pediatric Neurosci 1988: 14: 230-235.

10) Hamano S, Goto N, Nara T, Yamaguchi $K$ and Maekawa $K$. Development of the human mesencephalic trigeminal nucleus: a morphometric study. Dev Med Child Neurol 1990; 32:621-628.

11) Koningsmark BW. Methods for the counting of neurons. In Nauta WJH and Ebbesson SOE (ed): Contemporary Research Methods in Neuroanatomy. pp. 315-340. Springer, Berlin, 1970.

12) Nara T, Goto N and Yamaguchi K. Development of the human hypoglossal nucleus: a morphometric study. Dev Neurosci 1989; 11:212-220.

13) Nara T, Goto N, Nozaki H and Maekawa K. Development of the human facial nucleus: a morphometric study. No To Hattatsu 1989: 21:453-459 (in Japanese).

14) Nara $T$, Goto $N$, Nakac $Y$ and Okada A. Morphometric development of the human auditory system: ventral cochlear nucleus. Early Hum Dev 1993; 32:93-102.

15) Schimert J. Der Nervus intermedius und das Ganglion geniculi nervi facialis. Z Mikrosk Anat Forsch 1936: 39: 35-44. 


\section{Explanation of Figures}

\section{Plate I}

\begin{tabular}{c|c}
$1 \mathrm{a}$ & $1 \mathrm{~b}$ \\
\hline $2 \mathrm{a}$ & $2 \mathrm{~b}$ \\
\hline $3 a$ & $3 \mathrm{~b}$ \\
\hline $4 a$ & $4 b$
\end{tabular}

Fig. 1. Microscopic photos of the geniculate ganglion (LPH triple stain). The neuron in Fig. $1 \mathrm{~b}$ is an enlargement under high power view of the neuron indicated by an arrow in Fig. 1a.

Scale bar in Fig. $1 \mathrm{a}=100 \mu \mathrm{m}$, scale bar in Fig. $1 \mathrm{~b}=10 \mu \mathrm{m}$.

Fig. 2. Microscopic photos of the submandibular ganglion (LPH triple stain). The neuron in Fig. $2 b$ is an enlargement under high power view of the neuron indicated by an arrow in Fig. 2a. The magnification is the same as in Fig. 1.

Fig. 3. Microscopic photos of the fifth cervical spinal ganglion (K-B stain). The neuron in Fig. $3 b$ is an enlargement under high power view of the neuron indicated by an arrow in Fig. 3a. The magnification is the same as in Fig. 1.

Fig. 4. Microscopic photos of the superior cervical ganglion (K-B stain). The neuron in Fig. $4 \mathrm{~b}$ is an enlargement under high power view of the neuron indicated by an arrow in Fig. 4a. The magnification is the same as in Fig. 1. 

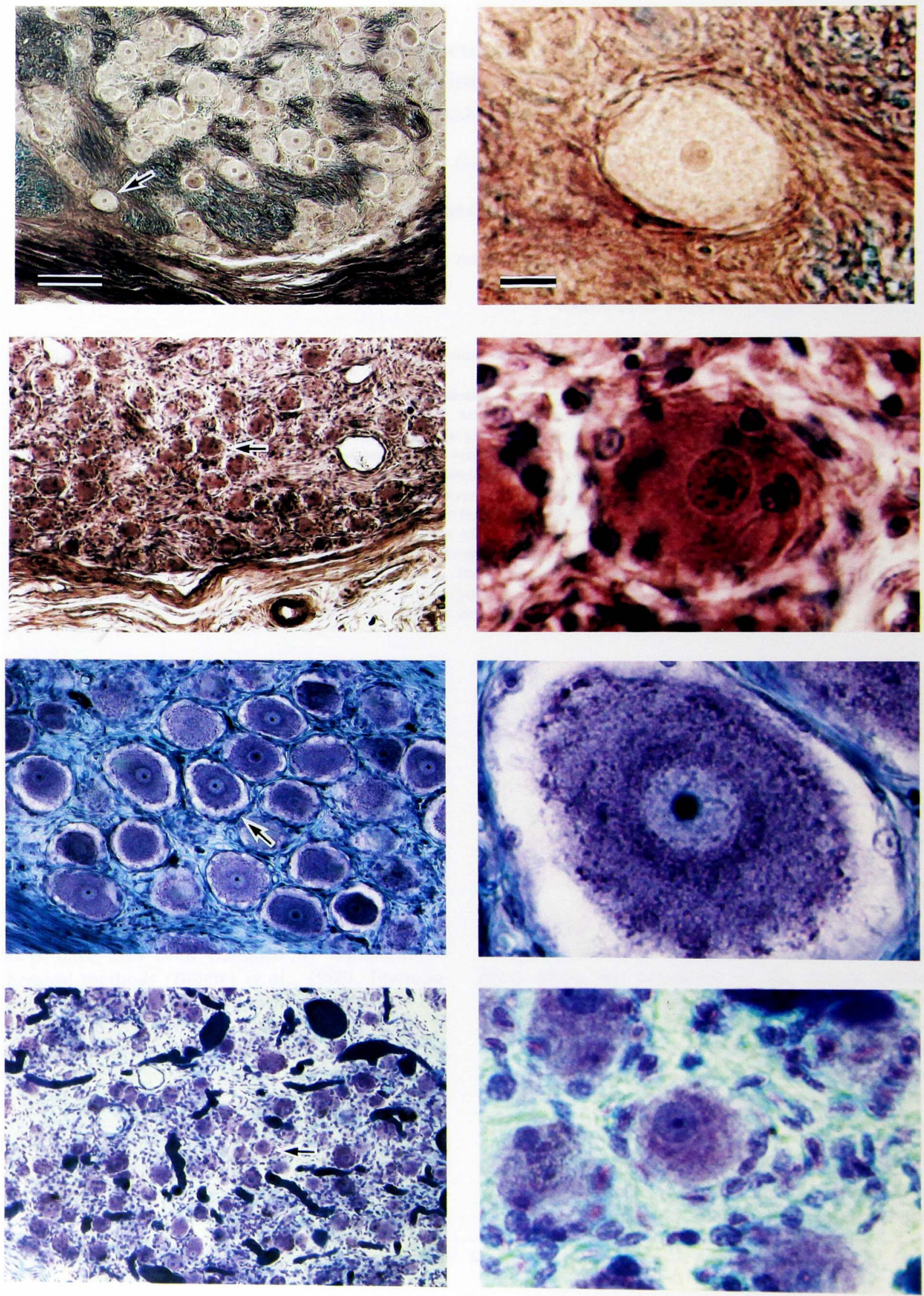